\title{
Efecto de la manzanilla (Matricaria camomilla) en el mildiu (Perenospora variabilis) de la quinua
}

\author{
Effect of chamomile (Matricaria camomilla) on downy mildew (Perenospora variabilis) of quinoa
} Efeito da camomila (Matricaria camomilla) sobre o míldio (Perenospora variabilis) da quinoa

\section{ARTÍCULO GENERAL}

Efraín Lindo Gutarra

Universidad Nacional del Centro del Perú, Huancayo - Perú

\section{Senin Marca Cano}

Universidad Nacional del Centro del Perú, Huancayo - Perú
Anghely Lapa Chanca

Universidad Nacional del Centro del Perú, Huancayo - Perú

Recibido 01 de Diciembre 2020 | Arbitrado y aceptado 26 de Diciembte 2020 | Publicado el 01 de Febrero 2021

\section{RESUMEN}

Con el objetivo de determinar la dosis en gramos de las flores de manzanilla para controlar los daños generados por el mildiu (Perenospora variabilis) en el cultivo de quinua se propuso trabajar con el extracto de las flores de manzanilla para evaluar su eficacia, contribuyendo así a la producción orgánica del cultivo de quinua. El estudio se basó en los efectos de la infusión de manzanilla en dosis de $50,100,150$ y $200 \mathrm{gr} /$ litro de agua con y sin adherente; y un Ridomil (control químico) se aplicaron a plantas de quinua y se evaluaron a los $5,8,10$ y 15 días después del control registrándose la incidencia y severidad en el tercio medio de la planta. Los datos registrados fueron en porcentaje de daño y los análisis estadísticos fueron contrastados en base el diseño completamente al azar con 3 repeticiones y la prueba de Tukey a un $95 \%$ de confiabilidad y para la eficacia se utilizó la fórmula de HendersonTilton, asimismo se calculó ABCP y ABCPr. Teniendo resultados favorables con el tratamiento de $150 \mathrm{gr}$. de manzanilla más adherente en el control de la incidencia de la enfermedad, y el tratamiento de 200 gr. de manzanilla más adherente en el control de la severidad de la enfermedad.

Palabras claves: Manzanilla, mildiu, quinua, incidencia, severidad.

\section{ABSTRACT}

In order to determine the dose in grams of chamomile flowers to control the damage caused by mildew (Perenospora variabilis) in the quinoa crop, it was proposed to work with the extract of chamomile flowers to evaluate its effectiveness, thus contributing to organic production of quinoa cultivation. The study was based on the effects of chamomile infusion in doses of 50,100,150 and $200 \mathrm{gr} /$ liter of water with and without adherent; and a Ridomil (chemical control) were applied to quinoa plants and were evaluated at 5,8,10 and 15 days after the control, recording the incidence and severity in the middle third of the plant. The data recorded were in percentage of damage and the statistical analyzes were contrasted based on the completely randomized design with 3 repetitions and the Tukey test at $95 \%$ reliability, and the Henderson-Tilton formula was used for efficacy. calculated $\mathrm{ABCP}$ and $\mathrm{ABCPr}$. Having favorable results with the treatment of 150 gr. of chamomile more adherent in the control of the incidence of the disease, and the treatment of $200 \mathrm{gr}$. of chamomile more adherent in the control of the severity of the disease.

Keywords: Chamomile, mildew, quinoa, incidence, severity.

\section{RESUMO}

Com o objetivo de determinar a dose em gramas de flores de camomila para controlar os danos causados pelo míldio (Perenospora variabilis) na cultura da quinoa, propôs-se trabalhar com o extrato de flores de camomila para avaliar sua eficácia, contribuindo assim para a produção orgânica de quinoa cultivo. $\mathrm{O}$ estudo baseou-se nos efeitos da infusão de camomila nas doses de 50,100,150 e 200gr/litro de água com e sem aderente; e um Ridomil (controle químico) foram aplicados em plantas de quinoa e avaliados aos 5, 8, 10 e 15 dias após o controle, registrando a incidência e severidade no terço médio da planta. Os dados registrados foram em porcentagem de dano e as análises estatísticas foram contrastadas com base no delineamento inteiramente casualizado com 3 repetições e o teste de Tukey com $95 \%$ de confiabilidade e para a eficácia foi utilizada a fórmula de Henderson-Tilton, da mesma forma a fórmula de Henderson-Tilton foi usado calculado ABCP e ABCPr. Tendo resultados favoráveis com o tratamento de 150 gr. de camomila mais aderente no controle da incidência da doença, e o tratamento de 200 gr. de camomila mais aderente no controle da gravidade da doença.

Palavras-chave: Camomila, míldio, quinoa, incidência, severidade. 


\section{Introducción}

La quinua (Chenopodium quinoa Willd), llamada también como el grano de oro, es un cultivo que en los últimos años ha incrementado su producción y consumo a nivel nacional e internacional, especialmente de forma orgánica. Según reportes del Ministerio de Agricultura, la producción de quinua en el Perú se incrementó de 28668 hectáreas en el 2003 a 56605 al 2014 (MINAGRI 2014). La SUNAT (2014), en el 2013, registró exportaciones de $18250 \mathrm{Tn}$, lo que generó ingresos por un total de US\$ 77,8 millones. Entre las enfermedades una de las más importantes es el mildiu causado por el oomycete Peronospora farinosa F. sp. Chenopodii, que en lugares donde hay alta humedad relativa y temperaturas entre 12 a $22^{\circ} \mathrm{C}$, puede causar grandes pérdidas (Alandia 1979, Otazú et al. 1976, Ochoa y Danial 1999). Choi et al. (2010) indicó que Peronospora farinosa posteriormente reconocida como, Peronospora variabilis Gäum es la especie que causa la enfermedad de mildiú en quinua. Generalmente, las condiciones ambientales con alta humedad favorecen el desarrollo del mildiu. La enfermedad se presenta en la mayoría de los lugares donde se cultiva la quinua, ello, por la gran diversidad genética del patógeno (Danielsen et al., 2000)

Con el aumento significativo del área de cultivo de la quinua en la última década los problemas ocasionados por el mildiú se han incrementado notablemente debido a la alta susceptibilidad de las variedades comerciales y a la existencia de ambientes favorables para su multiplicación. Con el fin de controlar a la enfermedad, los agricultores han utilizado tradicionalmente fungicidas en base a metalaxil. Sin embargo, los fungicidas son costosos, requieren múltiples aplicaciones, y finalmente, pueden ser superados por razas resistentes, ya que el patógeno es sexualmente recombinante (Aegerter et al 2002, Thomma 2003). Además, incrementan los costos de producción y afectan a la salud de los agricultores y al medioambiente (Gabriel 2010). Considerando lo anteriormente señalado es muy importante proponer alternativas de control a la enfermedad, por ello en esta investigación se propuso el control del mildiu con el extracto de las flores de manzanilla, para determinar una dosis que se pueda recomendar al agricultor.). Para Pauli, 2006 el extracto de manzanilla Debido a la bajo toxicidad del alfa-bisabolol el compuesto se cuestiona como un agente terapéutico y puede servir como un compuesto líder en el desarrollo de nuevos medicamentos antimicóticos. En otras 
investigaciones se reporta que el extracto de cola de caballo en cultivos de cebolla es eficaz en el control de mildiu seguido de la efectividad de extractos de tomillo y manzanilla (Gabela, 1999). Según Cubides (2013), el extracto de 8 aceites esenciales entre ellas de la manzanilla superó al ridomil para el control de incidencia y severidad de Peronospora variabilis en el bulbo de la cebolla.

\section{Materiales y Métodos}

La investigación se realizó en el año agrícola 2019-2020, en el paraje de Pirataco, de la localidad de Sicaya, provincia de Huancayo, Región Junín. Ubicado a 1200`45” de latitud sur, $75^{\circ} 17^{\prime} 00^{\prime \prime}$ de longitud Oeste, a una altitud de $3273 \mathrm{msnm}$.

Para la fase de campo se hizo la preparación del terreno, se prosiguió con la siembra de quinua variedad Hualhuas y las labores culturales como el deshierbo oportuno. Para la aplicación de del biopreparado de manzanilla se consideró desde el momento en que las plantas tengas dos hojas bien diferenciadas.

Se trabajó con 10 tratamientos:

T1 : Testigo absoluto.

T2 : 50 gramos manzanilla/litro de agua.

T3 : 100 gramos de manzanilla/litro de agua.

T4 : 150 gramos manzanilla/litro de agua.

T5 : 200 gramos manzanilla/litro de agua.

T6 : 50 gramos manzanilla/litro de agua con adherente.

T7 : 100 gramos manzanilla/litro de agua con adherente.

T8 : 150 gramos manzanilla/litro de agua con adherente.

T9 : 200 gramos manzanilla/litro de agua con adherente.

T10 : 2 gramos de Ridomil/litro de agua con adherente.

Los tratamientos se aplicaron a los 70 días después de la siembra y se evaluó a los $5,8,10$ y 15 días después del control. 


\section{Preparación del biopreparado de manzanilla}

Para preparar 1 lt. se utilizó: 25 gr. de flores de manzanilla (M. chamomilla) frescas o secas.

Se necesitó 1 lt. de agua caliente y 1 recipiente de 1 litro (preferentemente de plástico), 1 filtro. Después se colocó 25 gr. de flores de manzanilla, frescas o secas, en un litro de agua caliente dejando reposar media hora, filtrando y aplicando inmediatamente el preparado. Este mismo procedimiento se realizó para los demás tratamientos con el incremento de las flores de manzanilla para cada caso.

\section{Determinación de la eficacia relativa}

Para determinar la eficacia de fungicidas en campo, se utilizó la fórmula de Henderson-Tilton

$$
\% E=\left[1-\left(\frac{C a}{T a}\right)\left(\frac{T d}{C d}\right)\right](100)
$$

$\%$ E: Porcentaje de eficacia

$\mathrm{Ta}=$ Infestación en parcela tratada antes de aplicar el tratamiento.

$\mathrm{Td}=$ Infestación en parcela después de aplicar el tratamiento.

$\mathrm{Ca}=$ Infestación de parcela testigo antes de aplicar el tratamiento.

$\mathrm{Cd}=$ Infestación en parcela testigo después de aplicar el tratamiento.

\section{Área bajo la curva del progreso de la enfermedad (ABCPE)}

(Forbes, G; Pérez, W y Andrade-Piedra, P. 2014 y Campbell y Madden, 1990) reportan que el indicador como referencia del progreso de la enfermedad después de aplicar los tratamientos a los 70 días después de la siembra. Se utilizó la siguiente fórmula:

$$
A B C P E=\sum_{i=1}^{n} \frac{\left(X_{i+1}+X_{i}\right)}{2} *\left(T_{i+1}-T_{i}\right)
$$

Donde, 


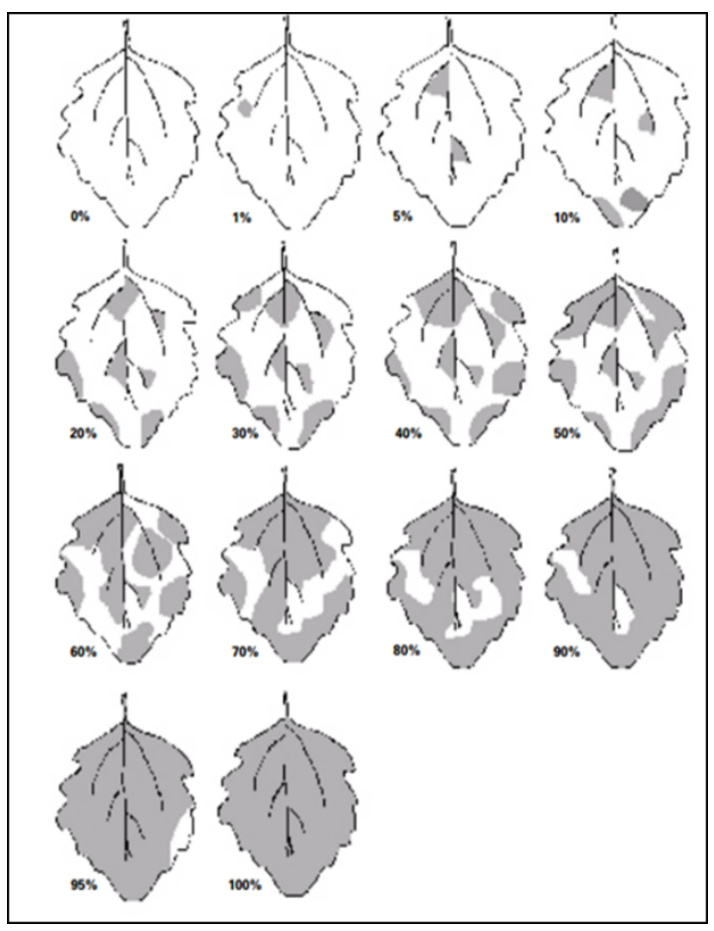

- Xi: Incidencia en la observación i.

- Ti+1 - Ti: Tiempo en días entre dos evaluaciones.

- $\mathrm{n}$ : número total de evaluaciones

\section{Área bajo la curva del progreso de la enfermedad relativa (ABCPEr)}

Este indicador determina el \% relativo de la infección del progreso de la enfermedad con la siguiente fórmula, (Forbes, G; Pérez, W y Andrade-Piedra, P. 2014).

$A B C P E_{r}=\frac{A B C P E}{U D-P D}$

UD: último día de la evaluación

PD: Primer día de la evaluación

\section{Recolección de datos}

Para el caso de la incidencia se consideró el tercio medio de la planta, observándose el número de hojas infestadas y de ahí se consideró el porcentaje de incidencia. 
Para el cado de severidad se tomó como referencia los métodos usados por Danielsen y Mulk (2004) y los grados de daños ocurridos en las hojas de acuerdo a la escala de la Dra. Teresa Ames, según el siguiente gráfico.

\section{RESULTADOS}

3.1. Efecto de infusión de manzanilla sobre la incidencia de la enfermedad

\subsubsection{Efecto a los 5 días después del control}

Tabla 1. Análisis de varianza del efecto de las flores de manzanilla en Peronospora variabilis en el cultivo de Chenopodium quinoa $W$. var. Hualhuas en el tercio medio (Incidencia) a los 5 días después del control, transformados a raíz cuadrada. SicayaHuancayo 2020.

\begin{tabular}{lccccc}
\hline $\begin{array}{c}\text { Fuente de } \\
\text { variabilidad }\end{array}$ & GL & CM & F & P & Sig, \\
\hline Bloques & 2 & 0,16 & 3,83 & 0,04 & NS \\
Tratamientos & 9 & 0,17 & 4,06 & 0,0056 & $*$ \\
Error & 18 & 0,04 & & & \\
Total & 29 & & & & \\
\hline Promedio & \multicolumn{7}{c}{ 5DDC } \\
\hline CV\% & 3,75 & & \\
\hline
\end{tabular}

En la Tabla 1, respecto a los cinco días después de la aplicación de la infusión de manzanilla se observa entre tratamientos una significación estadística por lo tanto entre los tratamientos hay una respuesta diferencial respecto a al porcentaje de incidencia de la enfermedad, el cuál es validado por un coeficiente de variabilidad del 3,75\% que significa que dentro de cada tratamiento hubo homogenidad de respuesta.

Tabla 2. Prueba de Duncan del efecto de las flores de manzanilla en Peronospora variabilis en el cultivo de Chenopodium quinoa $W$. var. Hualhuas en el tercio medio (Incidencia) antes y a los 5 días después del control. Sicaya-Huancayo 2020

\begin{tabular}{|c|c|c|c|c|c|c|c|}
\hline Nivel de probabilidad: 0,05 & & ADC & & & 5DDC & & \\
\hline Tratamientos & & & & & & & \\
\hline Producto y dosis de prueba & D.T. & D.o. & Sig. & D.T. & D.o. & & Sig \\
\hline T10: Ridomil & 5,55 & 30,77 & $\mathrm{a}$ & 5,71 & 32,67 & $\mathrm{a}$ & \\
\hline $\mathrm{T} 2: 50 \mathrm{~g}$ manzanilla $\mathrm{S}$ & 5,71 & 32,7 & a & 5,71 & 32,67 & $\mathrm{a}$ & \\
\hline T3: $100 \mathrm{~g}$ manzanilla $\mathrm{S}$ & 5,3 & 28,1 & a & 5,6 & 31,33 & & b \\
\hline T1:Testigo & 5,49 & 30,14 & a & 5,59 & 31,33 & & b \\
\hline T4: $150 \mathrm{~g}$ manzanilla $\mathrm{S}$ & 5,36 & 28,87 & a & 5,38 & 29.00 & $\mathrm{a}$ & b \\
\hline $\mathrm{T} 7$ : $100 \mathrm{~g}$ manzanilla $\mathrm{C}$ & 5,59 & 31,3 & a & 5,32 & 28,33 & $\mathrm{a}$ & b \\
\hline T9: $200 \mathrm{~g}$ manzanilla $\mathrm{C}$ & 5,45 & 29,73 & a & 5,32 & 28,33 & & b \\
\hline T8: $150 \mathrm{~g}$ manzanilla $\mathrm{C}$ & 5,6 & 31,4 & a & 5,26 & 27,67 & & $\mathrm{~b}$ \\
\hline T5: $200 \mathrm{~g}$ manzanilla $\mathrm{S}$ & 5,4 & 29,23 & a & 5,16 & 26,67 & & c \\
\hline T6: 50g manzanilla $\mathrm{C}$ & 5,61 & 31,47 & a & 5,03 & 25,33 & & $\mathrm{c}$ \\
\hline
\end{tabular}


En la Tabla 2, se observa antes de aplicar los tratamientos (ADC) una incidencia entre 28,10 hasta $32,70 \%$ es decir del tercio medio de la planta de quinua existió un promedio del $30 \%$ de hojas con daños de la enfermedad y después de la aplicación tanto de la infusión de manzanilla como el Ridomil estuvieron una incidencia entre 25,33 a $32,67 \%$.

\subsubsection{Efecto a los 8,10 y 15 días después del control}

Tabla 3. Análisis de varianza del efecto de las flores de manzanilla en Peronospora variabilis en el cultivo de Chenopodium quinoa $W$. var. Hualhuas en el tercio medio (Incidencia) a los 8,10 y 15 días después del control, transformados a raíz cuadrada. Sicaya-Huancayo 2020

\begin{tabular}{|c|c|c|c|c|c|c|c|c|c|c|c|c|c|}
\hline \multirow{2}{*}{$\begin{array}{c}\text { Fuente de } \\
\text { variabilidad }\end{array}$} & \multirow[b]{2}{*}{ GL } & \multicolumn{4}{|c|}{$8 \mathrm{DDC}$} & \multicolumn{4}{|c|}{ 10DDC } & \multicolumn{4}{|c|}{$15 \mathrm{DDC}$} \\
\hline & & $\mathrm{CM}$ & $\mathrm{F}$ & $P$ & Sig. & $\mathrm{CM}$ & $\mathrm{F}$ & $P$ & Sig. & $\mathrm{CM}$ & F & $P$ & Sig. \\
\hline Bloques & 2 & 0,02 & 0,25 & 0,78 & NS & 0,43 & 2,33 & 0,12 & NS & 0,25 & 1,74 & 0,204 & NS \\
\hline Tratamientos & 9 & 1,42 & 20,98 & $<0,0001$ & $* *$ & 3,76 & 20,15 & $<0,0001$ & $* *$ & 5,31 & 36,76 & $<0,0001$ & $* *$ \\
\hline Error & 18 & 0,07 & & & & 0,19 & & & & 0,14 & & & \\
\hline Total & 29 & & & & & & & & & & & & \\
\hline Promedio & & & 5 & 38 & & & 5 , & 68 & & & 5,0 & 63 & \\
\hline CV\% & & & 5 & 33 & & & 7, & & & & 6,7 & & \\
\hline
\end{tabular}

En la Tabla 3, respecto a los 8 días después de la aplicación de la infusión de manzanilla se observa una alta significación estadística entre tratamientos, por lo tanto, hubo un efecto directo del aceite de manzanilla en el desarrollo del mildiu de la quinua en las hojas y esta condición fue similar a medida que pasó el tiempo donde se observa a los 10 y 15 días después del control. Por otro lado, para diferenciar los tratamientos se utilizó la prueba de Duncan donde se observó que a los 8 días después del control sobresalió el Ridomil con un 10\% de incidencia que fue superior a los demás tratamientos. Para Calixtro, 2017. El mildiu se desarrolló en un $90 \%$ a los 104 días después de la siembra, lo que se relaciona con los datos obtenidos en la investigación.

Tabla 4. Prueba de Duncan del efecto de las flores de manzanilla en Peronospora variabilis en el cultivo de Chenopodium quinoa $W$. var. Hualhuas en el tercio medio (Incidencia) a los 8,10 y 15 días después del control, transformados a raíz cuadrada. Sicaya-Huancayo 


\begin{tabular}{|c|c|c|c|c|c|c|c|c|c|}
\hline \multirow{3}{*}{$\begin{array}{c}\text { Nivel de probabilidad: } \mathbf{0 , 0 5} \\
\text { Tratamie ntos } \\
\text { Producto y dosis de prue ba }\end{array}$} & \multicolumn{3}{|c|}{$8 D D C$} & \multicolumn{3}{|c|}{ 10DDC } & \multicolumn{3}{|c|}{ 15DDC } \\
\hline & Media & & & & & & & & \\
\hline & D.T. & D.o. & Sig. & D.T. & D.o. & sig. & D.T. & D.o. & Sig. \\
\hline $\begin{array}{l}\text { T10: Ridomil } \\
\end{array}$ & 3,16 & 10.00 & $\mathrm{a}$ & 2,63 & 7.00 & $\mathrm{a}$ & 2,29 & 5,33 & $\mathrm{a}$ \\
\hline $\mathrm{T} 9: 200 \mathrm{~g}$ manzanilla $\mathrm{C}$ & 4,5 & 20,33 & $\mathrm{bc}$ & 5,82 & 34.00 & $\mathrm{bc}$ & 5,56 & 31.00 & $\mathrm{~b} \mathrm{c}$ \\
\hline T8: 150g manzanilla C & 4,8 & 23.00 & $\mathrm{bc}$ & 5,62 & 32.00 & b & 5,22 & 27,67 & b \\
\hline $\mathrm{T} 7: 100 \mathrm{~g}$ manzanilla $\mathrm{C}$ & 4,33 & 23,33 & $\mathrm{bc}$ & 5,51 & 30,67 & b & 5,49 & 30,33 & $\mathrm{bc}$ \\
\hline T6: 50g manzanilla C & 5,26 & 27,67 & $\mathrm{~cd}$ & 6,05 & 36,67 & $\mathrm{bc}$ & 6,11 & 37,33 & $\mathrm{c}$ \\
\hline T5:200g manzanilla S & 4,76 & 22,67 & $\mathrm{~b} c \mathrm{c}$ & 6,23 & 39.00 & $\mathrm{bc}$ & 6,18 & 38,33 & $\mathrm{c}$ \\
\hline T4: $150 \mathrm{~g}$ manzanilla $\mathrm{S}$ & 5,16 & 26,67 & $\mathrm{~cd}$ & 6,03 & 36,33 & $\mathrm{~b} c \mathrm{c}$ & 5,89 & 34,67 & $\mathrm{bc}$ \\
\hline T3: $100 \mathrm{~g}$ manzanilla $\mathrm{S}$ & 5,22 & 27,33 & $\mathrm{~cd}$ & 6,05 & 36,67 & $\mathrm{bc}$ & 5,92 & 35.00 & $\mathrm{bc}$ \\
\hline T2: 50g manzanilla S & 5,38 & 29.00 & d & 6,23 & 39.00 & $\mathrm{~b} c \mathrm{c}$ & 6,21 & 38,67 & $\mathrm{c}$ \\
\hline T1:Testigo & 5,67 & 31,67 & d & 6,63 & 44.00 & $\mathrm{c}$ & 7,53 & 56,67 & d \\
\hline
\end{tabular}

Los tratamientos con manzanilla a las dosis de 200,150 y $100 \mathrm{~g}$ mezclado con adherente tuvieron una respuesta entre 23,33 a $20,33 \%$ de incidencia que a la vez entre ellos no tuvieron significación estadística. Por otro lado, los tratamientos con manzanilla con o sin adherente tuvieron similar respuesta a excepción del tratamiento sin adherente a una dosis de $50 \mathrm{~g}$ donde se obtuvo $29 \%$ de incidencia que fue estadísticamente similar al testigo que resultó con un $31,67 \%$. A los 10 días después del control se observa que ridomil tuvo una diferenciación alta respecto al control del mildiu en relación a los tratamientos con manzanilla sólo se observó un 7\% de incidencia mientras todos los tratamientos con o sin adherente utilizando las dosis de manzanilla tuvieron una incidencia entre 34 a 39\% que estadísticamente fueron similares, sin embargo fue mejor respecto al testigo donde se obtuvo una incidencia del 44\%. A los 15 días después del control se observa que ridomil tuvo una diferenciación alta respecto al control del mildiu en relación a los tratamientos con manzanilla sólo se observó un 5,33\% de incidencia mientras todos los tratamientos con o sin adherente utilizando las dosis de manzanilla tuvieron una incidencia entre 30,33 a $38 \%$ a excepción del tratamiento con adherente con $150 \mathrm{~g}$ de manzanilla que obtuvo un $27,67 \%$, sin embargo fueron mejores respecto al testigo donde se obtuvo una incidencia del $56,67 \%$.

Sobre estos resultados que es alentador en vista que se observa respecto al testigo existe una diferencia importante así mismo (Cubides, 2013), al evaluar la incidencia y severidad de P. destructor, y el número y el peso de bulbos cosechados. El extracto de manzanilla superó al Ridomil en el control de la incidencia y severidad de P. destructor sin diferencias estadísticas entre sí. Llama la atención que en la investigación de Mendez.et.Al. (2013), el extracto de manzanilla no haya tenido efecto, pero con esta investigación queda demostrado lo contrario.

3.2 Efecto de infusión de manzanilla sobre la severidad de la enfermedad 
Tabla 5. Análisis de varianza del efecto de las flores de manzanilla en Peronospora variabilis en el cultivo de Chenopodium quinoa W. var. Hualhuas en el tercio medio (\%severidad) a los 8,10 y 15 días después del control, transformados a raíz cuadrada. Sicaya-Huancayo

\begin{tabular}{|c|c|c|c|c|c|c|c|c|c|c|c|c|c|c|c|c|}
\hline \multirow{2}{*}{$\begin{array}{l}\text { Fuente de } \\
\text { variabilidad }\end{array}$} & \multicolumn{3}{|c|}{ 5DDC } & \multicolumn{5}{|c|}{ 8DDC } & \multicolumn{3}{|c|}{ 10DDC } & \multicolumn{5}{|c|}{ 15DDC } \\
\hline & GL & CM & $\mathbf{F}$ & $\mathbf{P}$ & Sig. & CM & $\mathbf{F}$ & $\mathbf{P}$ & Sig. & CM & $\mathbf{F}$ & $\mathbf{P}$ & Sig. & $\mathrm{CM}$ & F & Sig. \\
\hline Bloques & 2 & 0,2 & 11,51 & 0,0006 & NS & 0,03 & 0,85 & $<0,0001$ & $01 \mathrm{NS}$ & 0,09 & 2,25 & 0,134 & NS & 0,08 & $\begin{array}{ll}0,78 & 0,473\end{array}$ & NS \\
\hline Tratamientos & 9 & 0,71 & $41,53<$ & $<0,0001$ & 1 ** & 2,43 & 63,870 & 0,4419 & 9 ** & 2,71 & $64,91<$ & $<0,0001$ & *** & 2,1 & $21,1<0,0001$ & 1 ** \\
\hline Error & 18 & 0,02 & & & & 0,04 & & & & 0,04 & & & & 0,1 & & \\
\hline Total & 29 & & & & & & & & & & & & & & & \\
\hline Promedio & & & & 2,7 & & & & 86 & & & 3,63 & & & & 5,72 & \\
\hline $\mathrm{CV} \%$ & & & & 182 & & & & 6,8 & & & 5,62 & & & & 5,51 & \\
\hline
\end{tabular}

En la Tabla 5, se observa respecto a los 5,8, 10 y 15 días después de la aplicación de la infusión de manzanilla en las plantas de la quinua y para todos los casos resultó una alta significación estadística es decir el efecto de los tratamientos así como el testigo tuvieron resultados diferenciados en el grado de daño que ocasionaron esta enfermedad en las hojas de la quinua.

Respecto al bloque en todas las evaluaciones se registraron no significativo lo que significa que no hubo diferenciación en relación a los daños en la distribución de los tratamientos.

Respecto al coeficiente de variabilidad se observa para las 4 evaluaciones un indicador que oscila entre 2,7 a 5,72 el cuál determina que hubo alta una homogeneidad en cuanto al daño de severidad en las hojas de la enfermedad similar dentro de cada unidad experimental lo que permite afirmar sobre la severidad en las hojas del patógeno en general su distribución en el campo fue homogénea.

Tabla 6. Prueba de Duncan del efecto de las flores de manzanilla en Peronospora variabilis en el cultivo de Chenopodium quinoa $W$. var. Hualhuas en el tercio medio (Severidad (\%)) a los 5,8,10 y 15 días después del control, transformados a raíz cuadrada. Sicaya-Huancayo

\begin{tabular}{|c|c|c|c|c|c|c|c|c|c|c|c|c|c|c|c|}
\hline \multirow{3}{*}{$\begin{array}{l}\text { Nivel de probabilidad: } 0.05 \\
\text { Tratamientos } \\
\text { Producto y dosis de prueba }\end{array}$} & \multicolumn{3}{|c|}{$\mathrm{ADC}$} & \multicolumn{3}{|c|}{ 5DDC } & \multicolumn{3}{|c|}{$8 \mathrm{DDC}$} & \multicolumn{3}{|c|}{ 10DDC } & \multicolumn{3}{|c|}{ 15DDC } \\
\hline & & dia & & Me & & & Medi & & & & edia & & & edia & \\
\hline & D.T. & D.o. & & D.T. & D.o. & & D.T. & D.o. & & D.T. & D.o. & & DT. & D.o. & \\
\hline 10: Ridomil & 1,36 & 1,87 & $\mathrm{a}$ & 2,34 & $5,47 \mathrm{a}$ & & 1,91 & $3,67 \mathrm{a}$ & & 2,32 & $5,4 a$ & & 3,89 & $15,17 \mathrm{a}$ & \\
\hline & 1,3 & 1,83 & a & 2,35 & $5,53 \mathrm{a}$ & $a b$ & & 2,73 а & & 2,94 & 10,33 & $\mathrm{~b}$ & 5,28 & 28.00 & bc \\
\hline & 1,4 & 2,2 & a & 2,32 & $5,43 \mathrm{a}$ & & & 6,53 & bc & 2,94 & & $\mathrm{bc}$ & 5,53 & 30,67 & $\mathrm{bc}$ \\
\hline & 1,31 & 1.73 & a & $\begin{array}{l}2,59 \\
2.59\end{array}$ & 67. & $b c$ & & 8,33 & $\mathrm{~cd}$ & 3.41 & $\begin{array}{l}0,0,67 \\
11,67\end{array}$ & ${ }_{c d}$ & 5,59 & , & bc \\
\hline & 1,37 & 1,9 & a & 3,21 & 10,33 & d & 3,51 & 12,33 & $\mathrm{e}$ & 4,58 & 21.00 & $\mathrm{e}$ & 6,45 & 41,67 & \\
\hline & 1,42 & 2,03 & a & 2,35 & $5,53 \mathrm{al}$ & ab & 2,31 & 5,4 & $\mathrm{bc}$ & 3,1 & 9,67 & $\mathrm{bc}$ & 5,44 & $\begin{array}{l}29,0 / 7 \\
29,67\end{array}$ & \\
\hline & 1,4 & 2,2 & a & 2,38 & $5,7 \mathrm{al}$ & $a b$ & & $\begin{array}{l}6,4 \\
6,7\end{array}$ & bc & $\begin{array}{c}3,1 \\
3,11\end{array}$ & $\begin{array}{l}3,07 \\
9,67\end{array}$ & 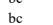 & 5,67 & 32,33 & bc \\
\hline & 1.4 & 2,2 & a & 2,6 & 7,23 & $\mathrm{c}$ & 05 & 9,33 & $\mathrm{~d}$ & 3,6 & 13.00 & $\mathrm{~d}$ & 5,91 & 35.00 & $\mathrm{~cd}$ \\
\hline T2: 50 & 1.4 & 2,0 & a & 3, & 9,83 & $\mathrm{~d}$ & 51 & 33 & $\mathrm{e}$ & 4,69 & 22.00 & $\mathrm{e}$ & 6,56 & 43.00 & \\
\hline T1 T1 Tstiog & 149 & 2,23 & 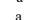 & 3,72 & 1,183 & e & 474 & 225 & $f$ & 54 & 2093 & $f$ & 688 & 47.33 & \\
\hline
\end{tabular}


En la Tabla 6 se observan los resultados del porcentaje de severidad antes y después del control y diferenciados mediante la prueba de Duncan entre los tratamientos. Antes de aplicar los tratamientos se observó que no hubo diferencias significativas en las unidades experimentales observándose de 1,87 a 2,83 de severidad que fue insignificantica el dalo promedio en hojas. A los cinco días después del control se observa que ridomil y las dosis de manzanilla con o sin adherente a las dosis de 150 y $200 \mathrm{~g}$ tuvieron resultados estadísticamente similares en relación al daño de las hojas respecto a la severidad que estuvo entre 5,43 a 5,7\% que fue altamente importante con la obstrucción en el desarrollo de la enfermedad, mientras a la dosis de $100 \mathrm{~g}$ de manzanilla con y sin adherente se obtuvo un daño en hojas entre un 6,7 a $7,23 \%$, mientras en el testigo se reportó un daño promedio de $13,83 \%$. Al octavo día después del control se resalta que la aplicación de manzanilla a la dosis de $200 \mathrm{~g}$ y con el ridomil tuvieron estadísticamente las mismas respuestas que osciló un daño entre 2,73 a 3,67\%, mientras en los tratamientos de $150 \mathrm{~g}$ con adherente y 200 y $150 \mathrm{~g}$ de infusión de manzanilla sin adherente tuvieron similar respuesta en daño que osciló entre 5,4 y 6,7\%, mientras usando manzanilla con y sin adherente con $50 \mathrm{~g}$ se obtuvo un daño que similar que registró para ambos $12,33 \%$ que fue menor al del testigo que registró $22,5 \%$.

3.3 Eficacia de los tratamientos respecto a la incidencia de la enfermedad

Tabla 7. Eficacia de los tratamientos respecto a la incidencia durante las evaluaciones al aplicar infusión de manzanilla para el control del mildiu en la quinua según Henderson-Tilton en porcentaje. Sicaya 2020

\begin{tabular}{lcccc}
\hline \multicolumn{1}{c}{ Tratamientos } & 5 & Días después del control \\
& 5 & 8 & 10 & 15 \\
\hline T2: $50 \mathrm{~g}$ manzanilla $\mathrm{S}$ & 3,89 & 15,6 & 18,3 & 37,1 \\
T3: $100 \mathrm{~g}$ manzanilla $\mathrm{S}$ & 0 & 7,44 & 10,61 & 33,76 \\
T4: $150 \mathrm{~g}$ manzanilla $\mathrm{S}$ & 3,37 & 12,08 & 13,8 & 36,13 \\
T5: $200 \mathrm{~g}$ manzanilla $\mathrm{S}$ & 12,22 & 26,19 & 8,6 & 30,26 \\
T6: $50 \mathrm{~g}$ manzanilla $\mathrm{C}$ & 22,57 & 16,32 & 20,18 & 36,91 \\
T7: $100 \mathrm{~g}$ manzanilla C & 12,93 & 29,06 & 32,88 & 48,46 \\
T8: $150 \mathrm{~g}$ manzanilla C & 15,23 & 30,29 & 30,19 & 53,13 \\
T9: $200 \mathrm{~g}$ manzanilla $\mathrm{C}$ & 8,33 & 34,92 & 21,66 & 44,54 \\
T10: Ridomil & 0 & 69,07 & 84,42 & 90,79 \\
\hline
\end{tabular}

En la Tabla 7 se observa la eficacia de la infusión de manzanilla respecto al ridomil, donde se observa a los 5 días después del control se registró para el ridomil un $0 \%$, mientras para la infusión de manzanilla a una dosis de $50 \mathrm{~g}$ con adherente un $22,57 \%$ esta 
respuesta se debió a que se evaluó en número de hojas del tercio medio de las plantas afectadas por la enfermedad es probable que hubo ciertos factores que influyó como por ejemplo el número de hojas tomadas en el tercio es relativo. Sin embargo a los 8 días después del control de los tratamientos se observa para el tratamiento con ridomil una eficacia del $69,07 \%$ que fue superior a todos los tratamientos con infusión de manzanilla y aquellos tratamientos superaron el 30\% fueron infusión de manzanilla con 150 y $200 \mathrm{~g}$ con adherente al décimo día después del control aquellos tratamientos que tuvieron adherente superaron el $20 \%$ de eficacia, mientras el ridomil logró un 84,42\% y a los 15 días después del control el ridomil registró un 90,79\% de eficacia y los tratamientos con manzanilla superaron el 33\% y el mayor se obtuvo a una dosis de $150 \mathrm{~g}$ con adherente, estos resultados variados en relación a los tratamientos y dosis se debe básicamente a la relatividad que se tomó el tercio medio donde se sumó al incremento del número de hojas sanas.

\subsection{Eficacia de los tratamientos respecto a la severidad de la enfermedad}

Tabla 8. Eficacia de los tratamientos respecto a la severidad (\%) durante las evaluaciones al aplicar infusión de manzanilla para el control del mildiu en la quinua según Henderson-Tilton en porcentaje. Sicaya 2020

\begin{tabular}{lcccc}
\hline \multicolumn{1}{c}{ Tratamientos } & \multicolumn{4}{c}{ Días después del control } \\
& 5 & 8 & 10 & 15 \\
\hline T2: $50 \mathrm{~g}$ manzanilla $\mathrm{S}$ & 23,43 & 40,96 & 19,19 & 2,13 \\
T3: 100g manzanilla $\mathrm{S}$ & 47,01 & 57,87 & 55,07 & 25,04 \\
T4: $150 \mathrm{~g}$ manzanilla $\mathrm{S}$ & 58,22 & 69,82 & 66,58 & 30,76 \\
T5: $00 \mathrm{~g}$ manzanilla $\mathrm{S}$ & 56,07 & 73,64 & 63,78 & 31,14 \\
T6: 50g manzanilla C & 12,33 & 35,68 & 15,97 & 0 \\
T7: 100g manzanilla C & 37,55 & 52,28 & 48,71 & 14,67 \\
T8: 150g manzanilla C & 60,2 & 70,58 & 70,04 & 34,32 \\
T9: 200 manzanilla C & 51,27 & 85,21 & 57,08 & 27,91 \\
T10: Ridomil & 52,83 & 80,55 & 78,04 & 61,78 \\
\hline
\end{tabular}

En la Tabla 8 se observa la eficacia de la infusión de manzanilla y el ridomil respecto a la severidad de la enfermedad en hojas de quinua del tercio medio, se observa a los 5 días después del control se registró un 60,2\% de eficacia a la dosis de la infusión de manzanilla a $150 \mathrm{~g}$ con adherente que superó al ridomil donde se obtuvo 52,83\%. Al octavo día después del control se observa una eficacia del $85,21 \%$ a una dosis de $200 \mathrm{~g}$ de infusión de flor de manzanilla con adherente que superó al ridomil donde se registró una eficacia de $80,55 \%$. Sin embargo a partir del décimo días existe una tendencia general 
con infusión de manzanilla a decrecer su eficacia y a los 15 días después del control la eficacia con infusión de manzanilla se logra una mejor eficacia obteniéndose con la dosis de $150 \mathrm{~g}$ una eficacia entre 27,91 a $30,76 \%$, mientras ridomil logra una eficacia del $61,78 \%$, esta respuesta se debe básicamente a que los aceites naturales como la infusión de la manzanilla tuvo efecto letal ante el hongo muy poco tiempo es decir que tiene una volatilidad efímera. Por ello (Cubides, 2013) en un experimento de extractos de vegetales: fueron evaluados en cuanto a su eficiencia en el control de P. destructor en cebolla de bulbo. Donde se aplicaron $(52,60,68,76,84,92$ y 100 días después del trasplante) cada 8 días, incluyendo un testigo químico Ridomil (metalaxil + mancozeb) (2 g/l) y el testigo absoluto. El extracto de manzanilla superó al Ridomil en el control de la incidencia y severidad de P. destructor sin diferencias estadísticas entre sí, por lo cual para nuestro experimento también es necesario la aplicación deberá ser con mayor frecuencia.

\section{5. Área bajo la curva del progreso de la enfermedad (incidencia)}

El propósito de evaluar este indicador es tener referencia como la enfermedad progresa realizando infestación o daño a la planta en un determinado tiempo, bajo estas consideraciones la infusión de manzanilla y el tratamiento con fungicida se aplicó a los 70 días de la siembra y se evaluó a los 5,8,10 y 15 días y en el gráfico 1, se observa que el testigo absoluto tuvo mayor susceptibilidad a la enfermedad es decir los daños se incrementaron con el tiempo superando el $50 \%$ con tendencias a incrementarse en el tiempo, mientras el tratamiento con ridomil a $2 \mathrm{~g}$ por litro tuvo una respuesta positiva donde los daños se redujeron en el tiempo que no superó el $10 \%$ esta disminución no significa que los síntomas registrados en el tercio medio de la planta haya desaparecido, sino que como la evaluación se ha realizado en el tercio medio en el crecimiento de la planta se ha incrementado el número de hojas sanas y ello ha contribuido a su disminución por un lado y el otro a que el producto químico ha controlado adecuadamente a la enfermedad no permitiendo su desarrollo en las hojas nuevas. Los tratamientos con manzanilla a los 15 días después de los tratamientos se observan un progreso similar de la enfermedad que a las mayores dosis con adherente hasta los $100 \mathrm{~g}$ se observa la detención de la enfermedad lo que se demuestra que la infusión de manzanilla si controla al mildiu, el cuál es una alternativa de suma importancia para obtener quinua orgánica en el valle. Risco (2014) encontraron incrementos de la enfermedad en los tratamientos 
estudiados entre los 55 y 60 días, explicando que esto probablemente se debe a que las plantas en este periodo emplean toda la energía para el crecimiento vegetativo que se expresa en una mayor masa foliar y que favorece la formación de microambientes favorables para el desarrollo del patógeno y una menor energía para emplear los mecanismos de defensa contra los estreses bióticos. De igual modo Kumar et al. (2006) señala que el valor más alto de ABCPE fue observado a los 65 días en un estudio realizado en la India con diferentes genotipos de quinua
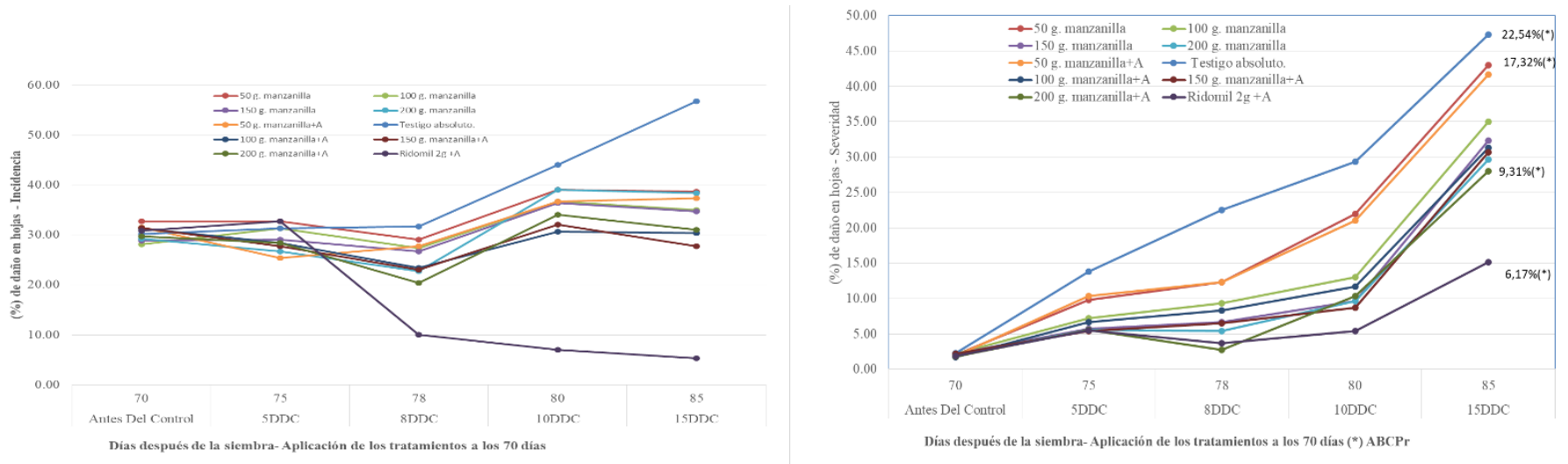

Gráfico 1. Área bajo la curva del progreso de la enfermedad (ABCPE) de la incidencia del mildiu en el tercio medio de la planta de quinua var. Hualhuas. SicayaHuancayo

\section{6. Área bajo la curva del progreso de la enfermedad (severidad)}

En el gráfico 2, se observa el progreso de la enfermedad en el grado de daño de las hojas de la quinua en tercio medio, el testigo absoluto tuvo una severidad mayor durante las evaluaciones al inicio se observó un 2\% y al final de la evaluación se registró acumulado sobre el 45\%, sin embargo realizando los cálculos sobre el área bajo la curva del progreso de la enfermedad relativa fue del $22,54 \%$, esto significa claramente que los daños que se genera a partir de la aplicación de los tratamientos en el caso del testigo se incrementan abruptamente, mientras con el producto ridomil se detiene los daños en las hojas donde se registró un $6,17 \%$ y los tratamientos con manzanilla se observan $9,31 \%$ para el caso de $200 \mathrm{~g}$ de infusión de manzanilla con adherente y el mayor daño se observó 
con la dosis de $50 \mathrm{~g}$ sin adherente obteniéndose $17,32 \%$ estos resultados demuestra que la infusión de manzanilla si controla la enfermedad y por lo cual, (Pauli, 2006). refiere que uno de los componentes de la infusión de la manzanilla interfiere con la biosíntesis de ergosterol a nivel de zimosterol y evitan la formación de fecosterol a partir de zymosterol que es el primer paso específico de hongos en la biosíntesis de ergosterol ello no se realiza en consecuencia el hongo colapsa.

Gráfico 2. Área bajo la curva del progreso de la enfermedad (ABCPE) de la severidad del mildiu en el tercio medio de la planta de quinua var. Hualhuas. SicayaHuancayo

\section{CONCLUSIONES}

La dosis de flores de manzanilla que tuvo más eficacia con respecto a la incidencia del hongo mildiu (Perenospora variabilis) en el cultivo de quinua es del tratamiento de 150 gr. con adherente, seguido de 100 gr y 200 gr con adherente. Con respecto a la severidad del hongo es el tratamiento de 200 gr con adherente.

Se determinó el número de aplicaciones para el control del mildiu de la quinua a partir de los 70 días después de la siembra es de 4 aplicaciones cada 5 días, teniendo en cuenta que el monitoreo de campo debe ser constante y que el control empieza desde la aparición de los primeros síntomas de la enfermedad.

\section{RECOMENDACIONES}

Replicar el mismo ensayo determinando un mayor número de aplicaciones para cada repetición con el objetivo de tener datos más exactos.

Realizar pruebas en donde se comparen otros extractos agrícolas de naturaleza como aceite de flor de manzanilla.

Realizar pruebas con dosis de $100 \mathrm{~g}$. de flor de manzanilla bajo el control preventivo al inicio de la enfermedad o cuando las condiciones climáticas son propicias para la enfermedad. 


\section{REFERENCIAS BIBLIOGRÁFICAS}

Aegerter BJ, Nuñez JJ, Davis RM. Detection and Management of Downy Mildew in Rose Rootstock. Plant Dis. 2002; 86(12):1363-1368.

Alandia S. Enfermedades. Páginas 137-144 in Quinua y kanihua. Cultivos Andinos. Tapia et al. (ed.). IICA, Bogotá, Colombia. 1979.

Calixtro, M. 2017. "Respuesta de 100 accesiones de quinua a la infección natural de mildiú (peronospora variabilis gäum) en el Valle del Mantaro tesis para opta el grado de ingeniero agronomo. Universidad nacional agraria la molina. 48.

CAMPBELL, C. L. AND MADDEN, L. V. 1990. Introduction to Plant Disease Epidemiology, Ed. Wiley. NY, USA. 532.CHOI, Y.; SEUNG, H.; SHIN, H. 2006. Re-consideration of Peronospora farinose infescting spinacia olareacea as distinct species, Peronospora effusa. Published by Elsevier Ltd. Seoul-Korea. 381-391 pp.

Cubides, P. (Enero-Junio de 2013). Evaluación de extractos de ocho especies vegetales en el control de mildeo velloso (Peronospora destructor Berk) en cebolla de bulbo (Allium cepa L.). Ciencia y Agricultura, 10(1), 81-89.

Danielsen, S; L. Munk y R. Nelson. 2000. AFLP and virulence markers for the characterization of Peronospora farinosa isolates from quinoa. Simposio 'Durable Disease Resistance', Nov. 28 - Dic. 1 2000, Ede - Wageningen, p. 38.

DANIELSEN, S.; MUNK, L. 2004. Evaluation of disease assessment methods in quinoa for their ability to predict yield loss caused by downy mildew. Crop Protection. 2004. Vol 23, Issue 3. 219-228.

Forbes, G., Pérez, W., \& Andrade- Piedra. (2014). Evaluación de la resistencia en genotipos de papa a Phytophthora infestans bajo condiciones de campo (Segunda ed.). Lima, Perú: Centro internacional de la papa.

Gabela, Paola. 1999. Control de Mildiú (Peronospora reductor) en cebolla "BOSUI" evaluando distancias de siembra y extractos vegetales. Tesis previa la obtención del título de Ingeniero Agrónomo. Quito-Ecuador 
Gabriel J. Documento marco: Estrategias y perspectivas del mejoramiento genético de papa (Solanum tuberosum L.) en Bolivia. ISBN: 978-99954-743-2-4, Fundación PROINPA, Cochabamba, Bolivia. 2010; 60 p.

KUMAR, A.; BHARGAVA, A.; SHUKLA S.; SINGH, H.B.; OHRI, D. 2006. Screening of exotic Chenopodium quinoa accessions for downy mildew resistance under mideastern conditions of India. Crop Protection. Vol 25, Issue 8. 879-889

Mendez, R.; Plata, G.; Gandarillas, A.; López, B.; Hinojosa, N. 2013. Desarrollo de biofungicidas en base a extractos de plantas para el control del Mildiu (Peronospora variabilis) de la quinua. Congreso científico de la quinua 14 y 15 de junio. La PazBolivia.

MINAG - OEEE (Direcciones Regionales y Subregionales de Agricultura). 2014. superficie de siembra y cosechada. Base de datos. s.p.

Ochoa J \& Danial D. Manejo de patógenos especializados en el mejoramiento genético de plantas para resistencia a enfermedades. In: Curso sobre aspectos técnicos en el manejo de los patosistemas de cultivos altos. 1999; 17-31 p.

Otazú V, Aguilar PC \& Canahua A. Resistencia en quinua (Chenopodium quinoa) al mildiú Peronospora effusa). Fitopatología. 1976; 11:47-49.

Pauli, A. (2006). Alfa-Bisabolol from chamomile-A specific ergosterol biosynthesis inhibitor? International Journal of Aromatherapy, 16(1), 21-25. Recuperado el 17 de Diciembre de 2019, de www.sciencedirect.com/science/article/pii/S0962456206000026

RISCO, A. 2014. Severidad de Peronospora variabilis GÄUM en Chenopodium quinoa Wiild Pasankalla como respuesta a aplicaciones de fungicidas sintéticos bioestimulantes. Tesis- Magister Scientiae en Fitopatología. Universidad Nacional Agraria La Molina. 91

SUNAT (Superintendencia Nacional de Administración Tributaria). 2014. Exportaciones de quinua a 37 mercados de destino. s.p.

Tomma BPHJ. Alternaria spp.: from general saprophyte to specific parasite. Molecular Plant Pathology. 2003; 4 (4): 225-236. 